\title{
Employee psychological reactions to micro-corporate social responsibility and societal behavior: A structural equation modeling analysis
}

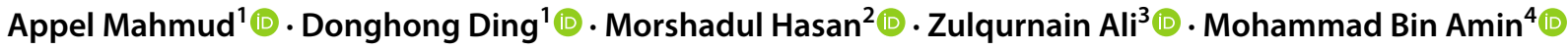

Accepted: 27 January 2022

๑) The Author(s), under exclusive licence to Springer Science+Business Media, LLC, part of Springer Nature 2022

\begin{abstract}
Employee psychological reactions to micro-corporate social responsibility (CSR) have recently been expanded in interdisciplinary management science research. The shreds of evidence in this regard are inconclusive, fragmented, and underdeveloped about how employee cognitive, behavioral, and affective dimensions of attitudes toward micro-CSR relate to each other and shape employee societal behavior (SB). In application of dual-process theories of attitude-behavior relations, we investigate the intra-relationships of perceived CSR-community (PCSRC; cognitive dimension of attitude), CSR engagement (CSRE; behavioral dimension of attitude), CSR positivity (CSRP; affective dimension of attitude). Also, we explore how these variables influence employee SB with a moderated-mediated model. Based on the opinions of 440 Bangladeshi employees as respondents, a structural equation modeling analysis confirmed the positive links from PCSRC to SB, PCSRC to CSRE, CSRE to SB, PCSRC to CSRP, CSRP to SB, and CSRP to CSRE. It also reported that CSRE mediated the relation between PCSRC and SB. It further examined that CSRP did not moderate the direct relation between PCSRC and SB, and the indirect relation between PCSRC and SB via CSRE at low, medium, or high employee CSRP. There are very crystal study implications that address policymakers to adopt CSR policy and its implementation strategies, accordingly, to employees' psychological reactions to micro-CSR.
\end{abstract}

Keywords Dual-process theories $\cdot$ Attitude-behavior relations $\cdot$ Micro (employee)-corporate social responsibility $\cdot$ Societal behavior · Bangladesh

\section{Introduction}

The contemporary stems of interdisciplinary management science research investigating the associations of employees psychological reactions such as commitment, emotion, pride, respect, honor, satisfaction, identity, engagement, attitude, and organizational and socially responsible behaviors such

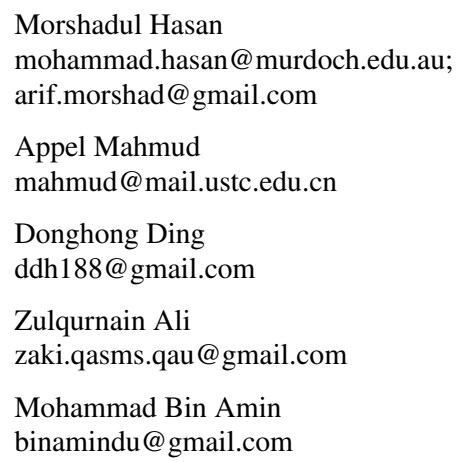


(SB; De Roeck \& Farooq, 2018) have been expanded in the micro-corporate social responsibility (CSR) studies (Barnett et al., 2020; Hill et al., 2021; Hur et al., 2021; Jurek \& Besta, 2021; Mahmud et al., 2021a, b; Maon et al., 2021; Moon et al., 2020). CSR as a broad area of study is popularly identified as "the commitment of business to contribute to sustainable economic development, working with employees, their families and the local communities"(World Business Council for Sustainable Development, 2001). In a similar vein, micro-CSR is designed as "the study of the effects and experiences of CSR on individuals (in any stakeholder group) as examined at the individual (shareholder, supplier, consumer, community, customer, or employee) level of analysis" (Rupp \& Mallory, 2015, p. 216). In a recent review, Barnett et al. (2020) argued that the micro-CSR domain reconceives itself as a science of design. It has already been an emerging research domain in the business, management, economics, and other social science fields (Gond \& Moser, 2021; Hur et al., 2021; Maon et al., 2021; Xiao et al., 2020).

A vital micro-cell of micro-CSR foundation exemplifies employees - the most dominating stakeholder group of a firm that is essential for enhancing brand image, business growth, social value, corporate reputation, better community development, and company-stakeholder relationships to attain its social, environmental, and economic goals as a whole sustainably (Aguinis \& Glavas, 2019; Chaudhary, 2020; Hur et al., 2021; Jurek \& Besta, 2021; Maon et al., 2021). As an insider stakeholder group, they have more credible company information than other stakeholders (Chaudhary, 2020; Hur et al., 2021; Mahmud et al., 2021b). They can sense and evaluate the motive for organizational engagement in CSR more profoundly based on a firm's values, beliefs, and history (Chaudhary, 2020; Hill et al., 2021; Hur et al., 2021). A recent meta-analysis of Zhao et al. (2020) revealed that to date, the micro (employee)-CSR research has primarily explored halo effects of employee attitudes toward CSR on traditional behavioral outcomes, such as corporate identity, organizational trust, workplace creativity, organizational justice, task performance, organizational commitment, job satisfaction, and turnover intention (also see Barnett et al., 2020; Rupp \& Mallory, 2015; Xiao et al., 2020 for reviews).

A few studies have investigated employee psychological reactions to micro-CSR and SB, although several researchers found positive associations between employees' attitudes to organizational CSR initiatives and socially responsible behaviors for the sustainable development of an economy (Hur et al., 2021; Mahmud et al., 2021b; Zhao et al., 2020). However, the shreds of evidence are inconclusive, fragmented, and underdeveloped about how employee psychological reactions to micro-CSR shape socially responsible behavior (notably SB; Jurek \& Besta, 2021; Maon et al., 2021; Moon et al., 2020). Also, we found limited studies that have explored how the components of employee attitudes such as the cognitive dimension of attitudes toward micro-CSR (notably perceived CSR-community [PCSRC]), the behavioral dimension of attitudes toward micro-CSR (notably CSR engagement [CSRE]), the affective dimension of attitudes toward micro-CSR (notably CSR positivity [CSRP]) are intra-related and how and when these components of employee attitudes restyle their SB in an emerging market (notably in Bangladesh) context.

Employee SB indicates "an individual employee's socially responsible actions and manners that support overall social welfare and community well-being even outside their organizational work context" (Mahmud et al., 2021b; Nazir et al., 2021). Employee SB and sustainable development of an emerging economy are positively related to fostering social, environmental, and economic growth, the standard of living, quality of progress, better community development, and overall well-being of society (Mahmud et al., 2021a; Zhao et al., 2020). Therefore, in the application of attitude-behavior relations (ABRs) models (Wicker, 1969) of dual-processing theories (DPTs) (Gawronski \& Creighton, 2013), the current study addresses some vital research gaps by exploring the links from PCSRC to SB, PCSRC to CSRE, CSRE to SB, PCSRC to CSRP, CSRP to SB, and CSRP to CSRE. Many micro-CSR studies lack the rigor of exploring the mediators and moderators of the CSR perception-employee outcome relationship (Hur et al., 2021). Expanding the possible mediators and moderators is essential in answering the unaddressed question of causality between CSR perceptions and employee outcomes (Hur et al., 2021). Therefore, it also examines the mediating role of CSRE in the relation between PCSRC and SB. It further investigates the moderating effects of CSRP in the direct relation between PCSRC and SB and indirect relation between PCSRC and SB via CSRE with a comparison at the low, medium, and high levels of CSRP. The four specific research questions of this current study are as follows. 1 . How does employee PCSRC affect SB and CSRE; CSRE influence SB? 2. How does employee PCSRC affect CSRP; CSRP influence SB; CSRP relate CSRE? 3. How does CSRE mediate the relations between PCSRC and SB? 4. When does the effect of CSRP strengthen the direct relationship between PCSRC and CSRE and the indirect relationships between PCSRC and SB via CSRE?

To test the proposed relations accordingly to research questions, we get survey data from Bangladesh. Bangladesh is now adorning the demographic dividend benefits (almost $65 \%$ of its population is working age, between 15 and 64) to demark its succeeding targets (Mahmud et al., 2020). With many prospects, Bangladesh is currently suffering from global issues (e.g., global warming, climate change, Rohingya refugee crisis, COVID-19 pandemic, increasing economic uncertainty, restricted access to quality education, 
poverty, hunger, environmental degradations, lack of good governance, and employee rights). These challenges are ushered by augmented pressure from various stakeholders to pay corporate resources to create business value, better community development, a safe environment, and societal progress in a globally responsible, accountable, and sustainable means (Mahmud et al., 2020; Mahmud et al., 2021b). Employee PCSRC, CSRE, and CSRP attitudes toward organizational socially responsible actions are dominant factors of a firm to donate corporate resources for restyling employee SB towards better community development and positive social change in Bangladesh (Mahmud et al., 2021b). It is ultimately a memorable occasion for Bangladeshi employees to be the co-partner of the regime and development partners to achieve its SDGs and accomplish its growth ambition of becoming an uppermiddle-income country by handling multiple economic challenges and social crises. Therefore, the inquiry of employee psychology such as attitudes, associations among the components of attitudes, and workforce psychological reactions to specific socially responsible activities (notably PCSRC) and SB is an urgently needed research agenda for policymakers and the academic world to upsurge the labor force in CSR involvement, gain investments in human capital, and raise productivity through increasing its vital stakeholder-business integration and contributing corporate resources as a voluntary business practice in Bangladesh (Girschik et al., 2020; Mahmud et al., 2020; Vizcano et al., 2021).

\section{Theory, Model, and Hypothesis}

\section{Theory and Model}

For the past four decades, many studies in social cognitions and socially responsible behaviors have been developed and directed by a class of social psychological theories popularly recognized as DPTs. DPTs have mainly been rooted in the prime assumption that human beings' mental processes, attitudes, and other psychological reactions to social actions are classified into two general classes: those have operated automatically in a social system, and those have functioned in a socially controlled environment (Gawronski \& Creighton, 2013). Early DPTs inclined to be domain-specific in that they absorbed particular social contextual phenomena such as attitude-behavior relationships. The most prominent phenomenon specific DPTs are ABRs models widely used to explore how people's particular attitudes influence their specific behaviors.

In this study, in applying ABR models of DPTs, we interpret employee attitudes as comprising three dimensions, specifically cognitive, behavioral, and affective attitudes that shape and change the SB of an employee. The cognitive dimension of employee attitude refers to "the employee perceptions of the organization's commitment to society" (Klimkiewicz \& Oltra, 2017). We proposed PCSRC as an example of cognitive dimension of employee attitude that is defined as "the firms' specific socially responsible activities to social welfare, such as charitable giving, donations, community development investments, social awareness programs, and collaboration with government officials and nongovernmental organizations for positive social change" (Mahmud et al., 2021b). The behavioral dimension of employee attitude represents to "the level of employee engagement in voluntary works, business ethics or CSR issues" (Klimkiewicz \& Oltra, 2017). We adopt CSRE as an example of behavioral dimension of employee attitude that is identified as "employee interest in CSR issues, such as business ethics, volunteering behaviors, willingness to deepen into eco-friendly actions, and active doers to link the society and business and communicate their mutual relationships" (Mahmud et al., 2021b). Finally, the affective dimension of employee attitude refers to "the employee assessment of critical social characteristics and CSR policy of an organization" (Klimkiewicz \& Oltra, 2017). We design CSRP as an example of affective dimension employee attitude that is recognized as "employee general feelings and positive attitudes toward a firm's better community developmentoriented socially responsible policies and actions that highlight employees' norms and manners of assessment of critical organizational culture, context, and CSR characteristics" (Mahmud et al., 2021b).

Expanding on how people's attitudes are shaped, changed, and directed, a class of DPTs of ABRs describes the processes through which individual attitudes guide their behavior (Gawronski \& Creighton, 2013). Thus, we attempt to investigate the links from PCSRC to SB, PCSRC to CSRE, CSRE to SB, PCSRC to CSRP, CSRP to SB, and CSRP to CSRE. ABR models have also been stimulated by frequent discussions about whether and to what extent people's attitudes influence their behavior (Gawronski \& Creighton, 2013). Therefore, we desire to explore the mediating mechanism of CSRE in the relation between PCSRC and SB. By shifting the focus from asking "Do attitudes guide behavior?" to the inquiry, "How do attitudes guide behavior?" ABR models of dual-process conceiving provided imperative insights into the conditions under which people's attitudes do or do not influence their discretionary behaviors (Gawronski \& Creighton, 2013). Thus, we construct a conceptual model to investigate the moderating effects of CSRP in the direct relation between PCSRC and SB and indirect relation between PCSRC and SB via CSRE with a comparison at the low, medium, and high levels of CSRP. Figure 1 represents the overall conceptual model to be empirically tested. 
Fig. 1 Conceptual model

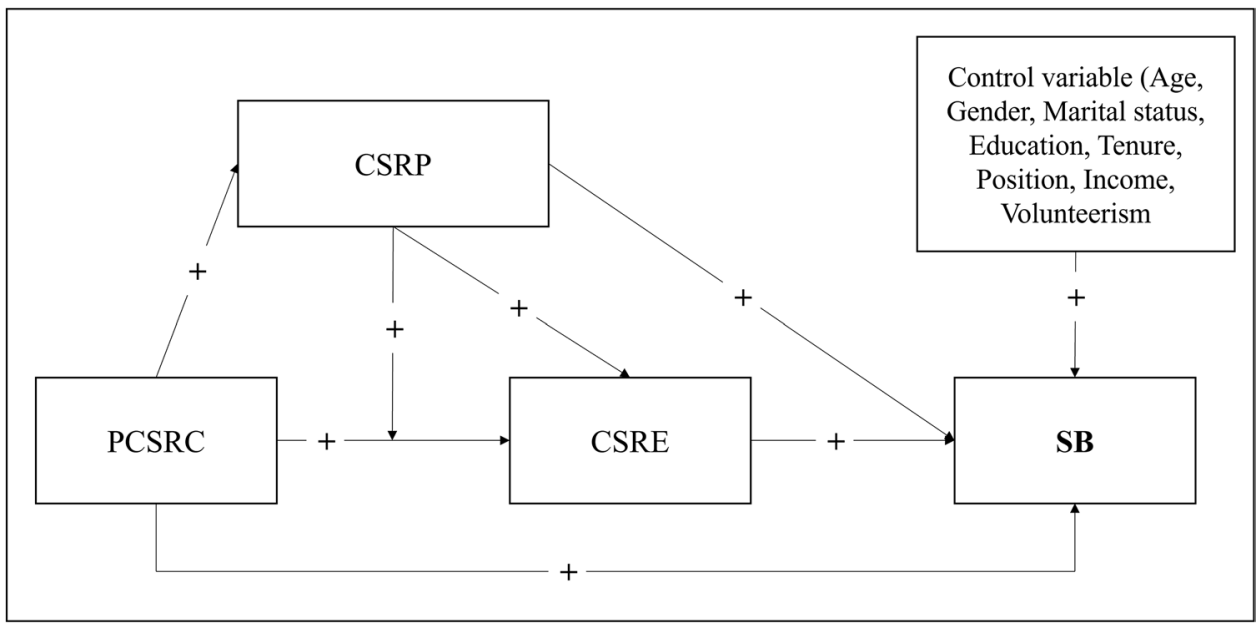

\section{Hypothesis Development}

\section{PCSRC, CSRE, and SB}

In the twentieth-first century, management science research is a time-driven shift from evaluating CSR's effects as a cumulation of all dimensions to account for a specific social turn (Mahmud et al., 2021b; Maon et al., 2021; Nazir et al., 2021). Given the recent global widespread attention and employees intensification in awareness of better community development and social responsibility, it is thinkable that the association of the cognitive, behavioral, and affective dimensions of employee attitudes toward organizational specific social activities (e.g., PCSRC) and their rational discretionary behavioral outcomes (e.g., SB) need to be further investigated in the research domain of micro-CSR and positive societal turns (Hur et al., 2021; Maon et al., 2021; Vizcaino et al., 2021).

The ABR models of DPTs also focus on how people's attitudes are formed, reshaped, and directed toward their discretionary behaviors (e.g., SB). Generally, a socially responsible employee emphasizes the effect of organizational social initiatives (e.g., PCSRC), the consequences of social activities, and the history of social actions rather than personal preferences and articulate decision-making passages (Mahmud et al., 2021b; Nazir et al., 2021). An employee is also more likely to involve in SB when his/her firm leads through its better community development-oriented CSR actions (i. e., PCSRC), credible informational cues about the status of contributory to better community development, and social well-being (De Roeck \& Farooq, 2018; Vizcaino et al., 2021). When an employee develops positive attitudes toward organizational better community development oriented-CSR statements or needs for overall social welfare (i.e., PCSRC), they use their firm's social information, information about past social contextual behaviors, and what civil society and other stakeholders think (De Roeck \& Farooq, 2018; Moon et al., 2020). The process of endorsing social needs or attitudes toward SB is affected by social information, the saliency of disclosures, and the significance of social statements, the stipulation to grow socially satisfactory and authentic justifications for social initiatives (Mahmud et al., 2021b; Nazir et al., 2021).

Employee social attitudes are vibrant antecedents of their psychological reactions to organizational CSR, especially PCSRC and positive social changes (Chaudhary, 2020; Mahmud et al., 2021a; Zhao et al., 2020). Practitioners and academics identify CSR attitudes as a valuable tool for addressing employees' social concerns and discretionary behaviors such as SB (Aguinis \& Glavas, 2019; Chaudhary, 2020). Hur et al. (2021) reported that a tradeoff occurs once employees shape positive attitudes that their firm aids or engages in better community development-oriented CSR (i.e., PCSRC) for positive social changes (e.g., charity-giving to community members, donations to vulnerable people, community development projects, and eco-friendly practices).

The extant literature in micro-CSR highlights that firms' specific social activities, especially PCSRCs, are considered a positive stimulus on employees' SB and their CSRE intentions (Hill et al., 2021; Moon et al., 2020). De Roeck and Farooq (2018) revealed that employee behavioral dimension of attitudes (i.e., CSRE) towards discretionary behaviors (i.e., SB) are natural outcomes of the consequence of insightful privilege of social initiatives and social, informational indications curbing from employee's organizational environment. They also reasoned that organizations' CSR novelties pay many prospects to form and change employees in CSRE in their work environment and community (e.g., donating to charity; investing labor, time, and money in social development projects; joining in the plan, design, promotion, and execution of CSR policies). Rupp et al. (2018) stated that employees are likely to rejoin unquestionably to intuitions 
of a firm stylish in better community development-oriented CSR and counter detrimentally to corporations whose business operations are alleged for harmful activities or negative CSR. In sum, previous research on employee attitudes and their socially responsible behavioral outcomes indicates that an employee who possesses solid ethical views on social obligations, business operations, and CSR practices will be more knotted in CSRE and SB than those who take only a profit maximization motive organizational social responsibility. Therefore, the overall research outcomes on DPTs and employee outcomes reveal that employee attitudes and SB are leading stubborn by the organizational proximal CSR context, especially PCSRC and CSRE. Thus, we propose the following Hypotheses.

HIa: PCSRC is positively related to employees' SB. HIb: PCSRC is positively related to employees' CSRE. H1c: CSRE is positively related to employees' $S B$.

\section{PCSRC, CSRP, SB, and CSRE}

PCSRC actions signify organizational social initiatives and social, informational signs that describe the desirable, supportive, and organizational social characteristics that also ultimately foster employees' feeling to support social credentials through their CSRP toward SB for better community development and social well-being (Aguinis \& Glavas, 2019; Gond \& Moser, 2021; Maon et al., 2021). A large body of contemporary evidence indicates that employee attitudes to organizational CSR policy and its execution practices affect employees' inclination to pay to, join in, and initiate positive social change movements (Chaudhary, 2020; Donia et al., 2019; Vizcaino et al., 2021). Gond and Moser (2021) found a meaningful connection between employee attitudes to CSR practices and meaningful work and job engagement. Rupp et al. (2018) reported that PCSRC suggestively improves employer-employee relations and employee affective dimension of attitudes (notably CSRP) is more likely to way for long-term benefits for business and society.

As a long-lasting discussion topic in the management and social science literature, a large body of evidence describes the attitude-behavior relationships with equivocal findings of human beings' social and environmental attitudes and their consequences on emotion, commitment, responsibility, and behaviors (Hur et al., 2021; Mahmud et al., 2020; Nazir et al., 2021). However, personal psychology, social-cognitive factors, and awareness of CSR have a sophisticated influence on the positive employee assessment of organizational CSR and their attraction and preservation of firms' social welfare motives (Jurek \& Besta, 2021; Mahmud et al., 2021a). Several micro-CSR researchers (Chaudhary, 2020; Hill et al., 2021) identified that the employee affective dimension of attitudes toward CSR, i.e., CSRP boosts company evaluation, brand reputation, and social image and a mode of uttering their prearranged identity into society. A firm's social initiatives (notably PCSRC) staple for its employees by conveying a signal to assess companies' fair-mindedness (Moon et al., 2020; Vlachos et al., 2017). PCSRC could disseminate positive social, informational signals to employees that positively upsurge their evaluations of the firm's reputation and involvement in better community development and overall social well-being (De Roeck \& Farooq, 2018; Hur et al., 2021; Vlachos et al., 2017).

The role of employees' positive perceptions (e.g., belonging, loyalty, pride) or the bearing of their negative attitudes (e.g., embarrassment, fear) for understanding how a specific CSR initiative (PCSRC) is perceived should also be considered in the attitude-behavior relationships investigations. Generally, employees are likely to react positively to perceptions of an employer engaging in socially responsible activities (e.g., community involvement, social welfare, or using environment-friendly materials in production) and retort negatively to a firm whose activities are alleged as socially irresponsible (Nazir et al., 2021; Rupp et al., 2018). Suppose socially responsible employees positively perceive the features of their organizational CSR policy, particularly development community development-oriented CSR initiatives (i.e., PCSRC). In that case, they are more likely to identify themselves with the employer and society so that they will value more be a part of the organization's CSR implementation and perform social work in their community (Mahmud et al., 2021b; Rupp et al., 2018). It signifies that employee swayed by organizational CSR policy may be more likely to identify with the CSR and business ethics concepts and engage themselves in CSR execution (Nazir et al., 2021; Vizcaino et al., 2021).

The existing literature on CSR-human resource management (HRM) outcomes academically and analytically designates that an employee who finds himself/herself engagement in organizational CSR execution (i.e., CSRE) as a part of the core value of work role meanings prompt to accomplish earnest duties and responsibilities such as work assignments and SB as his/her organizations bond and ethical viewpoints by gleaming such standards in the work environment and social culture (Jurek \& Besta, 2021; Rupp et al., 2018). Employees undertaking actions by engaging in CSR inventiveness allow them to extend their social prestige, organizational pride, and networking prospects in an informal setting, out-of-office, and making office alliances more supportive (Donia et al., 2019; Jurek \& Besta, 2021; Rupp et al., 2018). Consequently, if employees have a high level of mindfulness of social well-being (i.e., CSRP), they demonstrate many prosocial behaviors (notable $\mathrm{SB}$ ), resulting from even organization-person fit (Donia et al., 2019; Nazir et al., 2021). Employee CSRP is ever more substantial for firms' reputation and success, and in the same vein, it 
influences employees' SB and CSRE for overall social welfare as a whole (Donia et al., 2019; Gond \& Moser, 2021). Thus, we offer the following hypotheses.

H2a: Employee PCSRC is positively related to CSRP H2b: Employee CSRP is positively related to $S B$ and H2c: Employee CSRP is positively related to CSRE

\section{Mediating Condition of CSRE}

CSR researchers have premeditated many boundary conditions explaining how employees retort to cognitive, behavioral, and affective dimensions of attitudes (Donia et al., 2019; Hur et al., 2021). In line with this perceptive, micro-CSR scholars have argued that perceptions of the firms' social actions, such as its better community development oriented-social activities (i.c., PCSRC), can influence employees' social concerns and shape their discretionary behaviors (notably SB) through their involvement in social works (i.e., CSRE; Girschik et al., 2020; Maon et al., 2021). Chaudhary (2020) found that CSRE mediates CSR attributions and workers' creativity. Vlachos et al. (2017) reported that perceived CSR actions could raise employees' concerns about engaging in social works (discretionary behaviors) because CSR transmits social, informational reminders about performing in societal groups for better community development and social welfare.

Recent years have seen the dissemination of CSR initiatives that offer employees a chance to participate in missions with obvious positive social impact goals, often in partnership with government officials and non-government organizations (Bode \& Singh, 2018). Carmeli et al. (2017) reported that when employees observe that the firm is involved in activities that pay to improve environmental and societal issues and strengthen its affairs with the community, they are likely to perceive themselves as part of these initiatives and grow wisdom of gratification with this set of sustainable social actions. Employee CSRE is more often an apparatus to empower personnel to do "something more" on their assigned works and, in some cases, to yield worth for society and business (Girschik et al., 2020; Mahmud et al., 2020). Employees can act upon the central value and goal through CSR activities (Girschik et al., 2020; Moon et al., 2020). Employees who see organizational CSR contexts as the mechanisms to form encouraging social turns through their intervention are more likely to stem liveliness, psychological suppleness, a logic of implication, eagerness, and stimulus from individuals' CSRE (Gond \& Moser, 2021; Hur et al., 2021). In sum, this study predicts that the mediating role of employees' CSRE in PCSRC and their SB linkage might reasonably be expected from attitude-behavior relationships. Therefore, we propose Hypothesis 3 (H3) as follows.

H3: CSRE mediates the association between PCSRC and SB.

\section{Moderating Effect of CSRP}

CSRP is a strong predictor of the attitude-behavior relationships for assessing long-term employee CSR participation and extra-role behaviors (Girschik et al., 2020; Gond \& Moser, 2021). Most of the previous research in the CSR domain has tinted positive attitudinal and employees' behavioral outcomes of firms' engagement in CSR (Chaudhary, 2020; Jurek \& Besta, 2021). As social human beings, employees find meaningfulness in life through helping others improve their well-being due to the interactive nature of actions (Aguinis \& Glavas, 2019; Hur et al., 2021; Rupp et al., 2018). The positive assessment of organizational CSR initiatives forms employees' successive attitudes and behaviors toward their organization, communities, and society (Aguinis \& Glavas, 2019; Girschik et al., 2020). Employees evaluate CSR cues according to their observational behaviors and positive or negative attitudes toward CSR (Hur et al., 2021; Klimkiewicz \& Oltra, 2017). Therefore, employees' boldness to CSR plays a crucial part in understanding how personnel perceives CSR motions that ultimately influence their SB (Girschik et al., 2020; Nazir et al., 2021).

A firm's social actions (positive or negative) send employees vital information to judge their social motives and involve themselves in performing social actions according to its CSR implementation strategies. Bode and Singh (2018) found that many employees are concerned with involvement in the CSR initiatives even when CSRE requires individuals to sacrifice in a salary cut. Klimkiewicz and Oltra (2017) stated that social combination features, such as companies' alliance with government officials, non-government organizations, and cognizance of CSR practices, have a more potent stimulus on positive CSR evaluation and hereafter lure preservation of employees than personal factors. Gond and Moser (2021) claimed that employees with more vital CSRP experience more excellent social worth appropriate with firms more engaged in CSR, which leads to more tremendous positive social changes and organizational attractiveness. In sum, we assume that CSRP as a communal value strengthens the relation between organizational CSR initiatives (e.g., PCSRC) and employee CSRE directly and indirectly between organizational CSR initiatives (e.g., PCSRC) and individual discretionary behaviors (e.g., SB) through their CSRE. Thus, we propose Hypotheses $4(\mathrm{H} 4)$ and 5 (H5) as follows.

H4: CSRP moderates the linkage between PCSRC and CSRE, such that the association between PCSRC and CSRE is stronger when CSRP is high than low.

H5: CSRP moderates the indirect connection between $P C S R C$ and $S B$, such that the indirect impact of PCSRC on $S B$ via CSRE is strong when CSRP is high than low. 


\section{Methodology}

\section{Data and Sampling}

Based on the convincing sampling method, 440 Bangladeshi employees such as commercial bankers, textile and garment workers, and foreign wage earners were recruited through an online survey conducted from September 1 to December 31,2020 . A survey link was sent to each targeted employee's respective social media (e.g., Twitter, Facebook, and WhatsApp) with the concerned office's verbal approval. Firstly, we directed a pilot survey with 39 concerned employees to confirm the targeted respondents' knowledge of the survey items. The pilot survey participants did not encounter any severe issues while responding to the preliminary questionnaire. One professor, two doctoral students (currently researching micro-CSR), and two managerial employees were also requested to examine the survey items. We added their comments and recommendations to improve the respondents' understanding of the questionnaire items.

Secondly, we sent a survey link to 1000 targeted respondents. After giving a reminder (3 times) with an interval of two weeks, we obtained 472 responses with a response rate of $47.20 \%$. This response rate corresponded to the previous research on micro-CSR and employee attitudes and behavioral outcomes (Chaudhary, 2020; Mahmud et al., 2021b). We eliminated 32 responses due to the "straight-lining" problems. The final analysis was conducted on data collected from 440 respondents.

\section{Respondent Demographics}

We gathered the respondents' demographic information in a separate section of the survey form. Of the final sample (440), 51.4\% of the respondents were between 30 and 40 years old, $41.1 \%$ were between ages 40 and 50 years, only two respondents were below 30 but above 20 years old (.5\%), and $31(7 \%)$ respondents were 50 years old and above. The respondents' age group-based outcomes indicate that a young group of employees (30-50 years old) dominated the study outcome. In addition, $52.5 \%$ were men, and $47.5 \%$ were women respondents. Thus, a gender-based biasedfree study has both genders' almost equal participation in the survey. On the basis of the respondents' educational level, 163 (37\%) were graduate employees, and 277 (63\%) respondents held a master's degree or above. Thus, highly educated employees shared their obtained knowledge by participating in the survey. The present research outcomes were led by the married (66.1\%) versus unmarried (33.9\%) employees' viewpoints. Among the respondents, $47.5 \%$ had below 5 -year job experience, $43.6 \%$ were between 5 and 10 years of job experience, and only $39(8.9 \%)$ respondents had a job experience of above ten years. On the basis of the respondent's position in the organizational hierarchy, $242(55 \%)$ first-line managers' responses had more influence than 178 (40.5\%) middle-level employees, and only 20 (4.5\%) top-level managers' responses affected the present research outcomes. Most of the respondents had a high salary in the economic context of Bangladesh (i.e., $4.5 \%$ of the respondents were paid more than Taka100000 (Taka denotes Bangladesh currency), $18.9 \%$ of the respondents' salary was between Taka75000 and Taka100000, 30.7\% of the respondents had a salary of Taka $50,000-75,000$, and $45.7 \%$ of the respondents had a salary of between Taka 25,000-50,000. Almost $94 \%$ of the respondents were active volunteers in their community by sharing their income, time, and advice for better community development and social welfare.

\section{Measure}

We borrowed each construct's measurement scale in the current study from previous research. The respondents answered the questionnaire in Bangla (the national language of Bangladesh). The measurement scales were initially developed in English. Then, according to Hernández et al. (2020), we translated all the scale items utilizing the standard translation-back-translation method. All constructs projected in the present study were measured on a seven-point Likert scale (1 [strongly disagree] to 7 [strongly agree]).

\section{Perceived CSR Community (PCSRC)}

The PCSRC measurement scale was taken from De Roeck and Farooq (2018) and Mahmud et al. (2021b). We measured PCSRc with three items having a Cronbach's alpha (CA) of .87. Example items were, "My company gives adequate contributions to charities; My company supports NGOs actively working in problematic areas; My company contributes to campaigns and projects that promote the well-being of populations in the areas where it operates."

\section{Societal Behavior (SB)}

The SB measurement scale was taken from De Roeck and Farooq (2018) and Mahmud et al. (2021b). We measured employees' SB with four items having a CA of .89. Example items were, "I give adequate contributions to charities and donations; I usually donate blood for those who need it; I am involved in social and volunteer works that benefit my community; I engage myself in social and humanitarian causes and associations."

\section{CSR Engagement (CSRE)}

The CSRE measurement scale was taken from Klimkiewicz and Oltra (2017) and Mahmud et al. (2021b). We measured 
CSRE with six items having a CA of .90. Example items were, "I am interested in business ethics issues; Talking to my friends about work, I often go into ethical issues (e.g., regarding co-workers or managers' unethical behavior); When a company fails in ethical issues, I stop buying its products or resign from its services; I am an active volunteer; While shopping, I pay attention to the way the product was manufactured and transported; I would like to know more about CSR."

\section{CSR Positivity (CSRP)}

The CSRP measurement scale was taken from Klimkiewicz and Oltra (2017) and Mahmud et al. (2021b). We measured CSRP with six items having a CA of .91. Example items were, "Socially responsible companies operate in their interest and the interest of the whole society; CSR brings profits for organizations; I am skeptical toward organizations that define themselves as socially responsible*; If there were more socially responsible firms, then people would live better; Companies should not engage in solving social problems, as it is a state matter*; Including social and environmental issues in corporate policies enhances company competitiveness [*Reversed scale.].".

\section{Control Variables}

In line with the previous studies (Carmeli et al., 2017; Mahmud et al., 2021b), respondents' demographics (i.e., age, gender, marital status, education, tenure, position, income level, and volunteerism engagement) were used as control variables to explore the linkage of employees' perception on communityoriented CSR and SB.

\section{Analytical Strategy and Data Analysis}

The current study runs structural equation modeling (SEM) to test the proposed measurement model and structural relationships in Smart-PLS (Version 3). The SEM analytical strategy offers two outcomes simultaneously: factor and regression analyses (Hair et al., 2010; Hu \& Bentler, 1999). Hair et al. (2010) mentioned that SEM could manage the multifaceted proposed structural relationships proposed in the current research model. Thus, our study used this analytical strategy to handle the complex conceptual model having one moderator (CSRP) and one mediator (CSRP) in the relationship between PCSRc and SB by examining multiple related paths.

We conducted different data screening tests to ensure the findings' quality, reliability, and validity, such as multicollinearity, outliers, wrong coding, and missing values in SPSS. The findings do not confirm any of the issues in our sample. Non-response bias (NRB) was evaluated following Rogelberg and Stanton (2007). In doing so, we compared and contrasted early and late respondents $(n=39)$ using t-tests. The outcomes showed an insignificant association $(p>.05)$, indicating that NRB does not affect our study findings. We performed a common method variance (CMV) test due to the cross-sectional nature of our research design, following the criteria of Podsakoff et al. (2003). To do so, we used a full collinearity test suggested by Kock (2015) to ascertain if there is any CMV in our research instrument. Table 1 reveals the outputs of the full collinearity test. It has been found that all variance inflation factor (VIF) values turned to be lower than 3.3, indicating that $\mathrm{CMV}$ is not a big concern in our study.

\section{Results}

\section{Correlation Analysis}

Table 2 demonstrates the outcomes of intercorrelations. As expected, PCSRC was positively correlated with CSRE $(\mathrm{r}=.335, \mathrm{p}=.000)$ and SB $(\mathrm{r}=.351, \mathrm{p}=.000)$. Similarly,

Table 1 Full collinearity test

\begin{tabular}{|c|c|c|c|c|c|c|c|c|c|}
\hline & CSRE & CSRP & PCSRC & SB & & CSRE & CSRP & PCSRC & SB \\
\hline CSRE & & & & 1.256 & CSRE & & 1.201 & & \\
\hline CSRP & & & & 1.301 & CSRP & & & & \\
\hline PCSRC & & & & 1.209 & PCSRC & & 1.209 & & \\
\hline SB & & & & & SB & & 1.215 & & \\
\hline & CSRE & CSRP & PCSRC & SB & & CSRE & CSRP & PCSRC & SB \\
\hline CSRE & & & 1.301 & & CSRE & & & & \\
\hline CSRP & & & 1.258 & & CSRP & 1.21 & & & \\
\hline PCSRC & & & & & PCSRC & 1.253 & & & \\
\hline SB & & & 1.172 & & SB & 1.189 & & & \\
\hline
\end{tabular}

Source: Authors experiment [Note(s): CSRE $=\mathrm{CSR}$ engagement, $\mathrm{CSRP}=\mathrm{CSR}$ positivity; $\mathrm{PCSRC}=\mathrm{Per}-$ ceived CSR-Community, SB = Societal behavior] 
Table 2 Correlation

\begin{tabular}{lllll}
\hline Variables & CSRE & CSRP & PCSRC & SB \\
\hline CSRE & 1 & & & \\
CSRP & 0.425 & 1 & & \\
PCSRC & 0.335 & 0.375 & 1 & \\
SB & 0.347 & 0.304 & 0.351 & 1 \\
\hline
\end{tabular}

Source: Authors experiment [Note(s): Correlations are significant at $p<0.001 . \quad \mathrm{CSRE}=\mathrm{CSR}$ engagement, $\mathrm{CSRP}=\mathrm{CSR}$ positivity; PCSRC $=$ Perceived CSR-Community, $\mathrm{SB}=$ Societal behavior $]$

CSRE $(r=.347, \mathrm{p}=.000)$ was positively correlated with $\mathrm{SB}$. Furthermore, CSRP $(\mathrm{r}=.425, \mathrm{p}=.000)$ was positively correlated with CSRE. Hence, all these findings recognized our earlier structural relationships.

\section{Measurement Model}

We performed a confirmatory factor analysis (CFA) in PLSSEM to assess the proposed measurement model. The validity and reliability of the research scale were evaluated using the values of factor loading (FL), composite reliability (CR), and average variance extracted (AVE), as advised by Nunnally (1978) and Hair et al. (2010). CR values were higher than the cut-off $(<.70)$ criteria of reliable instruments, thus validating that all measures were reliable. Similarly, the FL and AVE values were higher than .70 and .50, respectively, indicating the research instrument's convergent validity (see Table 3). We also tested the discriminant validity for the study measures using Fornell-Larker Criterion (Fornell \& Larcker, 1981). We found that the square root of AVE for each construct (diagonal elements) was more significant than its correlation coefficients (off-diagonal elements), thereby

Table 3 Measurement model

\begin{tabular}{lllll}
\hline Constructs & Items & FL & CR & AVE \\
\hline CSRE & CSRE1 & 0.811 & 0.729 & 0.575 \\
& CSRE6 & 0.701 & & \\
CSRP & CSRP4 & 0.805 & 0.770 & 0.627 \\
& CSRP6 & 0.778 & & \\
PCSRC & PCSRC1 & 0.799 & 0.857 & 0.667 \\
& PCSRC2 & 0.879 & & \\
& PCSRC3 & 0.768 & & \\
SB & SB1 & 0.706 & 0.837 & 0.562 \\
& SB2 & 0.752 & & \\
& SB3 & 0.754 & & \\
& SB4 & 0.785 & & \\
\hline
\end{tabular}

Source: Authors experiment [Note(s): $\mathrm{FL}=$ Factor loading, $\mathrm{CR}=$ Composite reliability, and $\mathrm{AVE}=$ Average variance extracted, $\mathrm{CSRE}=\mathrm{CSR}$ engagement, $\mathrm{CSRP}=\mathrm{CSR}$ positivity; $\mathrm{PCSRC}=$ Perceived CSR-Community, $\mathrm{SB}=$ Societal behavior]
Table 4 Discriminant analysis (Fornell Larker Criterion)

\begin{tabular}{lllll}
\hline & CSRE & CSRP & PCSRC & SB \\
\hline CSRE & 0.756 & & & \\
CSRP & 0.429 & 0.791 & & \\
PCSRC & 0.336 & 0.374 & 0.816 & \\
SB & 0.341 & 0.304 & 0.350 & 0.750 \\
\hline
\end{tabular}

Source: Authors experiment [Note(s): CSRE $=$ CSR engagement, $\mathrm{CSRP}=\mathrm{CSR}$ positivity; $\mathrm{PCSRC}=$ Perceived $\mathrm{CSR}$-Community, $\mathrm{SB}=$ Societal behavior $]$

illustrating a solid discriminant validity of the study measures (See Table 4).

\section{Hypothesis Testing}

After the acceptance of the measurement model, we converted the measurement model into a structural model. Subsequently, we evaluated the values $\mathrm{R}$ square, $\mathrm{Q}$ square, and the significance of the paths coefficients. As evident from Appendix Tables 8 and 9, R square values for CSRE and CSRP .218 and .141, respectively, indicating that PCSRC and CSRE can explain $21.8 \%$ variance in CSRE; whereas, PCSRC and CSRP can explain $14.1 \%$ variance in CSRP. Besides, after controlling the effects of control variables, the $\mathrm{R}$ square value of SB turned to be .189 , indicating that CSRE can explain $18.9 \%$ variance in the SB, PCSRC, CSRP after controlling for the effects of Volunteerism, Gender, Tenure, Education, MS, Position, Age, Income.

To test the hypotheses accurately, we run the structural model twice, with and without control variables. As evident from Table 5, without control variables, all the direct paths from PCSRC to SB, PCSRC to CSRE, CSRE to SB, PCSRC to CSRP, CSRP to SB, and CSRP to CSRE turned to positive and statistically significant.

Table 6 demonstrates the results of hypotheses testing in the presence of control variables. It was found, in the presence of control variables, PCSRC was positively related to employees' SB ( $\beta=.223, S E=.056, t=3.979)$ and CSRE

Table 5 Testing hypotheses without control variables

\begin{tabular}{lllll}
\hline Paths & Std Beta & Std error & T Statistics & $P$ Values \\
\hline PCSRC $\rightarrow$ SB & 0.232 & 0.055 & 4.249 & 0.000 \\
PCSRC $\rightarrow$ CSRE & 0.209 & 0.048 & 4.308 & 0.000 \\
CSRE $\rightarrow$ SB & 0.217 & 0.059 & 3.694 & 0.000 \\
PCSRC $\rightarrow$ CSRP & 0.375 & 0.050 & 7.497 & 0.000 \\
CSRP $\rightarrow$ SB & 0.124 & 0.052 & 2.405 & 0.008 \\
CSRP $\rightarrow$ CSRE & 0.356 & 0.047 & 7.612 & 0.000 \\
\hline
\end{tabular}

Source: Authors experiment [Note(s): $\mathrm{PCSRC}=$ Perceived CSRCommunity, $\mathrm{SB}=$ Societal behavior; $\mathrm{CSRE}=\mathrm{CSR}$ engagement, $\mathrm{CSRP}=\mathrm{CSR}$ positivity 
Table 6 Testing hypotheses with control variables

\begin{tabular}{|c|c|c|c|c|c|c|}
\hline Hypotheses & & Paths & Std Beta & Std error & T Statistics & $P$ Values \\
\hline $\mathrm{H} 1 \mathrm{a}$ & & $\mathrm{PCSRC} \rightarrow \mathrm{SB}$ & 0.223 & 0.056 & 3.979 & 0.000 \\
\hline $\mathrm{H} 1 \mathrm{~b}$ & & $\mathrm{PCSRC} \rightarrow \mathrm{CSRE}$ & 0.209 & 0.049 & 4.290 & 0.000 \\
\hline \multirow[t]{4}{*}{$\mathrm{H} 1 \mathrm{c}$} & & $\mathrm{CSRE} \rightarrow \mathrm{SB}$ & 0.214 & 0.058 & 3.721 & 0.000 \\
\hline & $\mathrm{H} 2 \mathrm{a}$ & $\mathrm{PCSRC} \rightarrow \mathrm{CSRP}$ & 0.375 & 0.05 & 7.502 & 0.000 \\
\hline & $\mathrm{H} 2 \mathrm{~b}$ & $\mathrm{CSRP} \rightarrow \mathrm{SB}$ & 0.132 & 0.053 & 2.510 & 0.006 \\
\hline & $\mathrm{H} 2 \mathrm{c}$ & $\mathrm{CSRP} \rightarrow \mathrm{CSRE}$ & 0.356 & 0.047 & 7.507 & 0.000 \\
\hline \multirow{8}{*}{\multicolumn{2}{|c|}{ Effects of Control Variables on SB }} & Age $\rightarrow$ SB & 0.12 & 0.075 & 1.594 & 0.055 \\
\hline & & Education $\rightarrow \mathrm{SB}$ & -0.004 & 0.055 & 0.073 & 0.471 \\
\hline & & Gender $\rightarrow$ SB & 0.06 & 0.045 & 1.32 & 0.094 \\
\hline & & Income $\rightarrow$ SB & 0.161 & 0.081 & 1.991 & 0.023 \\
\hline & & $\mathrm{MS} \rightarrow \mathrm{SB}$ & -0.145 & 0.054 & 2.662 & 0.004 \\
\hline & & Position $\rightarrow \mathrm{SB}$ & 0.046 & 0.066 & 0.701 & 0.242 \\
\hline & & Tenure $\rightarrow$ SB & -0.147 & 0.086 & 1.707 & 0.044 \\
\hline & & Volunteerism $\rightarrow$ SB & 0.037 & 0.043 & 0.862 & 0.194 \\
\hline
\end{tabular}

Source: Authors experiment [Note(s): PCSRC $=$ Perceived CSR-Community, $\mathrm{SB}=$ Societal behavior, $\mathrm{CSRE}=\mathrm{CSR}$ engagement, $\mathrm{CSRP}=\mathrm{CSR}$ positivity; MS = Marital status]

$(\beta=.209, S E=.049, t=4.29)$, thereby supporting H1a and H1b. Similarly, CSRE $(\beta=.214, S E=.058, t=3.721)$ was positively related to employees' $\mathrm{SB}$, thereby supporting $\mathrm{H} 1 \mathrm{c}$. In addition, the paths from PCSRC and CSRP $(\beta=.375$, $S E=.05, t=7.502)$, from CSRP to $\mathrm{SB}(\beta=.132, S E=.053$, $t=2.51)$, and from CSRP and CSRE $(\beta=.356, S E=.047$, $t=7.507)$ were found positive and statistically significant. Hence, H2a, H2b, H2c were supported. Subsequently, we did not find any significant influence of the control variables, except for Income $(\beta=.161, S E=.081, t=1.991)$ and MS $(\beta=-0.145, S E=.054, t=2.662)$.

We investigated the mediation hypothesis (H3) that CSRE mediated the relationship between PCSRC and
SB. In doing so, we used Hayes and Preacher's (2010) bootstrapping approach (5000 iterations) at a 95\% confidence interval (CI) to measure the indirect influence. Bootstrap analysis is performed by constructing a large number of resamples $\mathrm{B}$ with an original sample size of $\mathrm{N}$, and each resample is constructed by sampling cases from data with replacements. The findings (Table 7) illustrated that the indirect effect $(\beta=.045, S E=.016, t=2.718$ ) of CSRE in the linkage between PCSRC and SB was significant, thus supporting $\mathrm{H} 3$.

Furthermore, we tested the moderating role of CSRP in the direct linkage between PCSRC and CSRE. In doing so, we utilized the product approach of Cohen and Cohen
Table 7 Testing mediation and moderation effects

\begin{tabular}{|c|c|c|c|c|c|}
\hline \multicolumn{6}{|c|}{ Testing indirect Effect/Mediation with Control Variables } \\
\hline Hypothesis & Indirect Path & Std Beta & Std error & T Statistics & $P$ Values \\
\hline $\mathrm{H} 3$ & PCSRC à CSRE à SB & 0.045 & 0.016 & 2.718 & 0.003 \\
\hline \multicolumn{6}{|c|}{ Testing Moderation Effects } \\
\hline Hypothesis & Interaction effect & Std Beta & Std error & T statistics & $P$ Values \\
\hline $\mathrm{H} 4$ & PCSRC $*$ CSRP à CSRE & 0.028 & 0.048 & 0.575 & 0.283 \\
\hline \multicolumn{6}{|c|}{ The Indirect Effect of PCSRC on SB through CSRE at Different Values of CSRP } \\
\hline CSRP & & Effect & SE & LLCI & ULCI \\
\hline Low & & 0.0468 & 0.022 & 0.0137 & 0.0988 \\
\hline Medium & & 0.054 & 0.0171 & 0.024 & 0.091 \\
\hline High & & 0.0612 & 0.021 & 0.0211 & 0.1025 \\
\hline \multicolumn{6}{|c|}{ Index of Moderated Mediation } \\
\hline Hypothesis & Moderator & Index & SE & LLCI & ULCI \\
\hline $\mathrm{H} 5$ & CSRP & 0.0072 & 0.0131 & -0.0256 & 0.0261 \\
\hline
\end{tabular}

$\mathrm{ULCI}=$ upper-level confidence interval 95\%, LLCI = lower level confidence interval 95\%, SE= Standard error]. 
(1983) and mean-centered our independent (PCSRC) and moderating (CSRP) variables. Then, we created the interaction term (multiplying independent and moderator) and ran the PLS-SEM moderation examination with CSRE (dependent variable). Consistent with our moderation hypotheses, the outcomes depicted that the interaction between PCSRC multiply CSRP and examining PLS-SEM moderation with CSRE found positive but insignificant $(\beta=.28, t=0.575, p=.283)$. Hence, moderation hypothesis $\mathrm{H} 4$ was not supported. Thus, we can conclude that the relationship between PCSRC and CSRE is not moderated by CSRP (See Table 7).

This study also investigated if CSRP moderates the mediating role of CSRE in the relationship between PCSRc and SB. Hence, we performed a moderated mediation analysis using model 7 of Process Macro (Hayes, 2015). Table 7 also shows the indirect effect of PCSRC on SB through CSRE at different values of CSRP. It can be observed that the path of PCSRC $\rightarrow$ CSRE $\rightarrow$ SB is statistically significant at low, medium, and high levels of CSRP. However, according to Hayes (2015), the index of moderated mediation is regarded as an actual test of moderated mediation (Table 7).

Evidence for the moderation of the indirect effect (PCSRC $\rightarrow$ CSRE $\rightarrow$ SB) can be found in the bootstrap confidence interval for the index of moderated mediation does not include zero in between (Hayes, 2015). As shown in Table 7, the index of moderated mediation is .0072, and the $95 \%$ percent bias-corrected bootstrap confidence interval based on 5000 bootstrap sample ranges from -.0256 to .0261 . Since the confidence interval for the index of moderated mediation includes zero in between, we can conclude that CSRP does not moderate the indirect effect of PCSRC on SB through CSRE. Therefore, H5 was not supported in this study.

\section{Discussion and Conclusion}

\section{Discussion}

Previous studies on CSR have primarily focused on the effects of total CSR and stakeholders' behavioral outcomes and their participation in CSR and extended prior findings (Hill et al., 2021; Hur et al., 2021; Vizcaino et al., 2021). They also demonstrated that even in an ongoing debate, employees could more favorably perceive micro-CSR initiatives as a vital internal stakeholder group. In this line-up, the current study explores underexplored micro-CSR initiatives (i.e., PCSRC) in applying popular social-psychological DPTs of attitudebehavior relationship. It tests the effects of employee PCSRC on their SB and CSRE; the influence of CSRE on SB. The current study's outcomes report that PCSRC is positively related to SB and CSRE. It also shows that CSRE is positively related to SB. These outcomes are consistent with those of De Roeck and Farooq (2018), Klimkiewicz and Oltra (2017), and Mahmud et al. (2021b). They also found positive relations between social welfare oriented-CSR and environmental degradation oriented-CSR employee socially responsible behaviors (social and green behaviors of employees). Our findings report that PCSRC allows the creation and strengthening of firms' social relationships, lessens negative feelings related to a suspected bad relationship between a company and its key stakeholders, and enhances employee SB and CSRE intentions.

This study also reports that PCSRC is positively related to CSRP; CSRP is positively related to SB and CSRE. Previous studies overlooked these links among employee attitudes and their behavioral perceptions. This study states that the employee affective dimension of attitude toward CSR (e.g., CSRP) plays a significant role in understanding how they perceive specific CSR signals and ultimately influence CSR-based employer attractiveness. Therefore, employees are expected to be positively reactive to employers appealing in socially accountable activities and negatively reactive to employers' perceived actions as environmentally and socially irresponsible.

This study also finds an answer of how, when, and under what condition employees' perceptions of organizational specific social activity (e.g., CSR-community) turn their specific socially responsible behaviors (e.g., SB) with a mediator (CSRE) and a moderator (CSRP) variable. The mediating mechanism is a pressing issue to justify organizational CSR activities and relational behavioral outcomes (Chaudhary, 2020; Hur et al., 2021). The current study reports that CSRE mediates the relation of PCSRC and SB consistents with De Roeck and Farooq (2018), Klimkiewicz and Oltra (2017), and Mahmud et al. (2021b). They stated that when employees observe their firms' CSR activities as promising, they are likely to carry more liveliness to exertion and parade better assignation of CSR and assigned work. In the previous outline perspectives, the study reports that employees as human beings find meaningfulness in life by improving others' well-being by their CSRE or intentions of CSRE; CSRE directly influences employer branding; particularly, employees who pose a sophisticated assignation with CSR demonstrate a developed CSR-based employer attractiveness than those who expose a low assignation with CSR.

The ABR models of DPTs are popularly taken to test contingency effects between CSR perceptions and employees' behavioral outcomes in the CSR-HRM research domains (Chaudhary, 2020; De Roeck \& Farooq, 2018). The current study reports that CSRP does not moderate the direct relationship between PCSRC and 
CSRE and the indirect relationship between PCSRC and SB via CSRE at the low, medium, or high employee CSRP. However, Klimkiewicz and Oltra (2017) revealed that employees' CSRP is directly related to employer attractiveness. CSR is exceptionally significant for company selection to employees who distinguish CSR positively. Mahmud et al. (2021b) found CSRP moderates the direct relationship between PCSRC and CSRE and the indirect relationship between PCSRC and SB via CSRE such that these relationships are significant at the high level of employees' CSRP and insignificant when employees' CSRP is low.

\section{Theoretical Contribution}

CSR-sophisticated employees are also more prospective to dispensation career if the organization flops in CSR acquiescence (Aguinis \& Glavas, 2019; Hur et al., 2021; Nazir et al., 2021). Employee organizational identification intermediates the affiliation amid employees' perception of micro-CSR (i.e., CSR-community, and CSR-environment) and discretionary behaviors (i.e., societal and green behaviors; De Roeck \& Farooq, 2018; Hill et al., 2021). Girschik et al. (2020) found that employee CSR perceptions have an enormously positive effect on their attitude toward CSR directly and indirectly (via work meaningfulness) among workforces with firmer morality beliefs and frailer centrality moral identity. Thus, similar to previous works, the current empirical faiths extend the understanding of organizational micro-CSR's strategic contributions into business value and society view by examining the links from PCSRC to SB, PCSRC to CSRE, CSRE to SB, PCSRC to CSRP, CSRP to SB, and CSRP to CSRE with a mediating (CSRE) boundary and a moderating condition in the application of DPTs of attitude-behavior relation in a newly emerging economy context (notably Bangladesh).

The findings support all the hypothesized relationships built on the ABR models of DPTs. Thus, the current study extends DPTs in the literature on the CSR-HRM domains. The current study's novel contribution is to find CSRE as a new intervening mechanism in CSR's contemporary literature. It is also treated as the first of the study of three dimensions of attitudes in the contemporary literature on the micro-CSR-HRM domains, such as cognitive, behavioral, and affective dimensions of attitudes within three microconcept views such as micro (employee)-level analysis, micro-CSR area (PCSRC), and micro-level work setting (SB). The current study bridges the gaps between developing and developed nations as CSR study contexts (dominated by the developed economies) and between macro and micro levels of CSR literature (the larger volume of CSR literature is occupied with macro-CSR).

\section{Implication for Policy Making}

The current study's findings indicate that a specific microCSR dimension (i.e., PCSRC) is more likely to create employees' particular socially responsible behavior (i.e., SB). Employees have a pivotal role in spinning organizational CSR investments into social welfare and business value (Aguinis \& Glavas, 2019; De Roeck \& Farooq, 2018; Hur et al., 2021). When a firm engages in CSR due to its intrinsic value to help society, it is a signal that employees will also be praised well, and the firm will not turn unscrupulously (Chaudhary, 2020; Nazir et al., 2021). As CSR performance boosts employer attractiveness for prospective and existing employees, companies that target to increase their social performance by shaping employees' discretionary behaviors through their engagement in CSR need to certify that firms' CSR policy is designed within the matching line of employees' desire to organizations for helping better community development and overall social welfare (Donia et al., 2019; Hur et al., 2021; Klimkiewicz \& Oltra, 2017). Therefore, firms' CSR policy and implementation strategies should be cautiously planned to gain a good result in the anticipated discretionary behaviors.

In the present study, more than half (55\%) of the respondents are first-line workers (prime CSR ambassadors) who are directly related to customer services, and $40.5 \%$ are positioned as middle-level employees who make the liaison between the top official and first-line management personnel who confirm the proposed structural relationships. Thus, CSR managers can use CSR as a valuable strategic managerial tool to increase mid-level employees and first-line workers positive reactions to organizational CSR because when they observe their firm's CSR initiatives are to help better community development and overall social welfare, they feel a sense of pride in and respect with the society for their respective organizational identity (De Roeck \& Farooq, 2018; Nazir et al., 2021).

HR managers should aim to advance socially responsible HRM by arranging seminars, workshops, training, and knowledge-sharing opportunities to develop employees' positive attitudes toward their firm's CSR initiatives into business value and social welfare. To do so, HR managers can announce CSR awards and labels for employees' active engagement in CSR activities that publicly recognize their firm's efforts and their participation in society, which will flow the internal organizational pride and external prestige 
in their organizational membership. PCSRC is positively related to employees' discretionary behaviors and CSRE, and CSRP is also positively related to CSRE; thus, CSR managers should adopt a CSR policy that addresses the social needs for better community development and positive social turns of Bangladesh (Mahmud et al., 2020).

\section{Conclusion}

The micro (employee)-CSR study that explores the association of employee psychology and organizational CSR initiatives that can foster social turns is still under focused and left aside in the emerging markets contexts and literature on the CSR-HRM domains (Hill et al., 2021; Hur et al., 2021; Rupp et al., 2018). The current study aims to bridge the gap between macro and micro-foundations by investigating the intra-relationships among employee cognitive, behavioral, and affective dimensions of attitudes and their impacts on SB under the ABR models of DPTs in a newly emerging market (notably Bangladesh) context. In the twenty-first century, CSR research is a time-driven shift from evaluating CSR's effect as a cumulation of all dimensions to account for the influence of a specific CSR subdimension on positive social change (Maon et al., 2021; Nazir et al., 2021). As a win-win-win strategy, CSR is now more effectively utilized for business growth, societal progress, and employees' well-being (Girschik et al., 2020; Mahmud et al., 2021a). As firms' insiders, employees are a powerful patron group; their responses to organizational CSR exertion are related to understanding the societal good created by CSR inventiveness (Chaudhary, 2020; Vizcaino et al., 2021). Our study expands DPTs in the CSR literature by revealing the positive associations of PCSRC, CSRE, CSRP, and SB with the mediating role of CSRE and the boundary condition of CSRP (although the moderation conditions are not supported). Therefore, firms should plan CSR policy and its impersonation strategy accordingly to employees' psychological reactions to organizational CSR, such as attitudes, emotions, feeling, engagement, and positivity (Hur et al., 2021; Jurek \& Besta, 2021; Vizcaino et al., 2021).

The generalizability of the current study's findings can be questionable because a few employees have participated in the survey from a South Asian emerging economy (Bangladesh) having multiple economic, social, and environmental crises. As such, further research can replicate this conceptual model in other national, cultural, and economic (developing and developed) contexts to corroborate our findings and conclusion. Another limitation is that this study is crosssectional self-reported data, which may produce a CMV bias. Although we assessed CMV that was minimum in the current research findings, future studies should perform on longitudinal design to check our model over time and generalize our findings. We collected data on a single period; thus, we suggest future research to project longitudinal and experimental inquiries to investigate further impressions of specific CSR dimensions and employee discretionary behaviors in the micro-CSR domain.

Beyond methodological limitations, social scientists can extend the micro-CSR literature by exploring other stakeholders (e.g., community, customer, and shareholder), micro-CSR perceptions, and SB. Our model can be extended by examining how firms' specific social, informational cues (e.g., CSR-environment) can eventually interact with employees' work engagement and discretionary behaviors (e.g., green behaviors). Potential researchers can also expand our model on other mediating mechanisms (e.g., respect, pride, and justice) that might decode stakeholders' perceptions on specific CSR dimensions into discretionary behaviors in the workplaces and out of the office. Although CSRP as a moderating variable does not support the assumption, potential moderators (e.g., public value awareness) that might resonate with the credibility and authenticity of firms' assignation in CSR should also be considered to comprehend the specific micro-CSR-SB relationships well.

Beyond variable(s) selection, an emerging body of literature needs to examine the effect of a firm's specific social activities on sustainability with potential CSR scholars' extensive attention to engross in sensing latent social outcomes of CSR initiative, not just economic value (Hur et al., 2021; Nazir et al., 2021). Lastly, if CSR is not rooted in social value, it might be alleged as greenwashing, which can shape employees' negative CSR experience, resulting in deviance and disengagement (Girschik et al., 2020; Mahmud et al., 2021b). Thus, future research might address the conditions under which perceived CSR can simultaneously move to the win-win-win outcomes of social welfare, employee well-being, and business value.

\section{Appendix}

Table 8 Note: This data is mandatory. Please provide

\begin{tabular}{ll}
\hline $\begin{array}{l}\text { Endogenous Con- } \\
\text { structs }\end{array}$ & R Square \\
\hline CSRE & 0.218 \\
CSRP & 0.141 \\
\hline
\end{tabular}


Table 9 Note: This data is mandatory. Please provide

\begin{tabular}{|c|c|c|c|c|c|c|c|c|c|}
\hline \multicolumn{10}{|c|}{ R Square of SB } \\
\hline \multirow[t]{2}{*}{ Model } & \multirow[t]{2}{*}{$\mathrm{R}$} & \multirow[t]{2}{*}{ R Square } & \multirow{2}{*}{$\begin{array}{l}\text { Adjusted R } \\
\text { Square }\end{array}$} & \multirow{2}{*}{$\begin{array}{l}\text { Std. Error of the } \\
\text { Estimate }\end{array}$} & \multicolumn{5}{|l|}{ Change Statistics } \\
\hline & & & & & R Square Change & F Change & df1 & df2 & Sig. F Change \\
\hline 1 & $.186^{\mathrm{a}}$ & 0.034 & 0.016 & 0.99283 & 0.034 & 1.921 & 8 & 431 & 0.055 \\
\hline 2 & $.472^{\mathrm{b}}$ & 0.223 & 0.203 & 0.89375 & 0.189 & 34.618 & 3 & 428 & 0.000 \\
\hline
\end{tabular}

a. Predictors: (Constant), Volunteerism, Gender, Tenure, Education, MS, Position, Age, Income

b. Predictors: (Constant), Volunteerism, Gender, Tenure, Education, MS, Position, Age, Income, CSRE, PCSRC, CSRP

Data Availability All data generated during and/or analyzed during the current study are available from the corresponding author on reasonable request.

\section{Declarations}

Ethics Approval The questionnaire and methodology for this study was approved by the concerned offices.

Consent to Participate Informed consent was obtained from all individual participants included in the study.

Conflicting Interests There is no conflict of interest among the authors.

\section{References}

Aguinis, H., \& Glavas, A. (2019). On corporate social responsibility, Sensemaking, and the search for meaningfulness through work. Journal of Management, 45(3), 1057-1086. https://doi.org/10. $1177 / 0149206317691575$

Barnett, M. L., Henriques, I., \& Husted, B. W. (2020). Beyond good intentions: Designing CSR initiatives for greater social impact. Journal of Management, 46(6), 937-964. https://doi.org/10.1177/ 0149206319900539

Bode, C., \& Singh, J. (2018). Taking a hit to save the world? Employee participation in a corporate social initiative. Strategic Management Journal, 39(4), 1003-1030. https://doi.org/10.1002/smj.2762

Brieger, S. A., Anderer, S., Fröhlich, A., et al. (2020). Too much of a good thing? On the relationship between CSR and employee work addiction. Journal of Business Ethics, 166, 311-329. https://doi. org/10.1007/s10551-019-04141-8

Carmeli, A., Brammer, S., Gomes, E., \& Tarba, S. Y. (2017). An organizational ethics of care and employee involvement in sustainability-related behaviors: A social identity perspective. Journal of Organizational Behavior, 38(9), 1380-1395. https://doi.org/10. $1002 /$ job. 2185

Chaudhary, R. (2020). Corporate social responsibility and employee performance: A study among Indian business executives. International Journal of Human Resource Management, 31(21), 27612784. https://doi.org/10.1080/09585192.2018.1469159

Cheema, S., Afsar, B., Al-Ghazali, B. M., \& Maqsoom, A. (2020). How employee's perceived corporate social responsibility affects employee's pro-environmental behaviour? The influence of organizational identification, corporate entrepreneurship, and environmental consciousness. Corporate Social Responsibility and Environmental Management, 27(2), 616-629. https://doi. org/10.1002/csr.1826
Chia, A., Kern, M. L., \& Neville, B. A. (2020). CSR for happiness: Corporate determinants of societal happiness as social responsibility. Business Ethics- A European Review, 29(3), 422-437. https://doi.org/10.1111/beer.12274

Cohen, J., \& Cohen, P. (1983). Applied multiple regression correlation analysis for the behavioral sciences. Erlbaum.

De Roeck, K., \& Farooq, O. (2018). Corporate social responsibility and ethical leadership: Investigating their interactive effect on employees' socially responsible behaviors. Journal of Business Ethics, 151, 923-939. https://doi.org/10.1007/s10551-017-3656-6

Ding, H., Lin, X., \& Su, W. (2021). Employee strengths use and innovative behavior: A moderated mediation model. Chinese Management Studies, 15(2), 350-362. https://doi.org/10.1108/ CMS-05-2019-0191

Donia, M. B. L., Ronen, S., Tetrault Sirsly, C. A., et al. (2019). CSR by any other name? The differential impact of substantive and symbolic CSR attributions on employee outcomes. Journal of Business Ethics, 157, 503-523. https://doi.org/10.1007/ s10551-017-3673-5

Fornell, C., \& Larcker, D. F. (1981). Evaluating structural equation models with unobservable variables and measurement error. Journal of Marketing Research, 18(1), 39-50. https://doi.org/10.1177/ 002224378101800104

Gawronski, B., \& Creighton, L. A. (2013). Dual-process theories. In D. E. Carlston (Ed.), The Oxford handbook of social cognition (pp. 282-312). Oxford University Press. https://psycnet.apa.org/ record/2013-34444-014

Girschik, V., Svystunova, L., \& Lysova, E. I. (2020). Transforming corporate social responsibilities: Toward an intellectual activist research agenda for micro-CSR research. Human Relations, 1-30, article available in OnlineFirst,. https://doi.org/10.1177/ 0018726720970275

Gond, J.-P., \& Moser, C. (2021). Critical essay: The reconciliation of fraternal twins: Integrating the psychological and sociological approaches to 'micro' corporate social responsibility. Human Relations, 74(1), 5-40. https://doi.org/10.1177/0018726719864407

Hair, J. F., Black, W. C., Babin, B. J., Anderson, R. E., \& Tatham, R. (2010). Multivariate data analysis (7th ed.). Pearson.

Hayes, A. F. (2015). An index and test of linear moderated mediation. Multivariate Behavioral Research, 50(1), 1-22. https://doi. org/10.1080/00273171.2014.962683

Hayes, A. F., \& Preacher, K. J. (2010). Quantifying and testing indirect effects in simple mediation models when the constituent paths are nonlinear. Multivariate Behavioral Research, 45(4), 627-660. https://doi.org/10.1080/00273171.2010.498290

Hernández, A., Hidalgo, M. D., Hambleton, R. K., \& Gómez-Benito, J. (2020). International test commission guidelines for test adaptation: A criterion checklist. Psicothema, 32(3), 390-398. https://doi.org/10.7334/psicothema2019.306

Hill, A. D., Johnson, S. G., Greco, L. M., O’Boyle, E. H., \& Walter, S. L. (2021). Endogeneity: A review and agenda for the 
methodology-practice divide affecting micro and macro research. Journal of Management, 47(1), 105-143. https://doi. org/10.1177/0149206320960533

Hu, L., \& Bentler, P. M. (1999). Cut-off criteria for fit indexes in covariance structure analysis: Conventional criteria versus new alternatives. Structural Equation Modeling: A Multidisciplinary Journal, 6(1), 1-55. https://doi.org/10.1080/107055199095401 18

Hur, W. M., Moon, T. W., \& Choi, W. H. (2021). The role of job crafting and perceived organizational support in the link between employees' CSR perceptions and job performance: A moderated mediation model. Current Psychology, 40, 3151-3165. https://doi. org/10.1007/s12144-019-00242-9

Jurek, P., \& Besta, T. (2021). Employees' self-expansion as a mediator between perceived work conditions and work engagement and productive behaviors. Current Psychology, 40, 3048-3057. https:// doi.org/10.1007/s12144-019-00241-w

Kim, A., Kim, Y., \& Han, K. (2019). A cross level investigation on the linkage between job satisfaction and voluntary workplace green behavior. Journal of Business Ethics, 159, 1199-1214. https://doi. org/10.1007/s10551-018-3776-7

Klimkiewicz, K., \& Oltra, V. (2017). Does CSR enhance employer attractiveness? The role of millennial job seekers' attitudes. Corporate Social Responsibility and Environmental Management, 24(5), 449-463. https://doi.org/10.1002/csr.1419

Kock, N. (2015). Common method bias in PLS-SEM: A full collinearity assessment approach. International Journal of e-Collaboration, 11(4), 1-10. https://doi.org/10.4018/ijec.2015100101

Mahmud, A., Ding, D., Kiani, A., \& Hasan, M. M. (2020). Corporate social responsibility programs and community perceptions of societal Progress in Bangladesh: A multimethod approach. SAGE Open, 10(2), 1-17. https://doi.org/10.1177/2158244020924046

Mahmud, A., Ding, D., \& Hasan, M. (2021a). Corporate social responsibility: Business responses to coronavirus (COVID-19) pandemic. SAGE Open, 11(1), 1-17. https://doi.org/10.1177/21582 44020988710

Mahmud, A., Ding, D., \& Ali, Z. (2021b). An investigation of employee perception of micro-corporate social responsibility and societal behavior: A moderated-mediated model. International Journal of Emerging Markets, Ahead-of-Print (ahead-of-print). https://doi. org/10.1108/IJOEM-02-2021-0266

Maon, F., Swaen, V., \& De Roeck, K. (2021). Corporate branding and corporate social responsibility: Toward a multi-stakeholder interpretive perspective. Journal of Business Research, 126, 64-77. https://doi.org/10.1016/j.jbusres.2020.12.057

Moon, T. W., Youn, N., Hur, W. M., et al. (2020). Does employees' spirituality enhance job performance? The mediating roles of intrinsic motivation and job crafting. Current Psychology, 39, 1618-1634. https://doi.org/10.1007/s12144-018-9864-0

Nazir, O., Islam, J. U., \& Rahman, Z. (2021). Effect of CSR participation on employee sense of purpose and experienced meaningfulness: A self-determination theory perspective. Journal of Hospitality and Tourism Management, 46, 123-133. https://doi.org/10. 1016/j.jhtm.2020.12.002
Nunnally, J. C. (1978). Psychometric theory. McGraw-Hill.

Podsakoff, P. M., Mackenzie, S. B., Lee, J., \& Podsakoff, N. P. (2003). Common method biases in behavioral research: A critical review of the literature and recommended remedies. Journal of Applied Psychology, 88(5), 879-903. https://doi.org/10.1037/0021-9010. 88.5.879

Rice, D. B., Young, N. C. J., \& Sheridan, S. (2021). Improving employee emotional and behavioral investments through the trickle-down effect of organizational inclusiveness and the role of moral supervisors. Journal of Business and Psychology, 36, 267-282. https://doi.org/10.1007/s10869-019-09675-2

Rogelberg, S. G., \& Stanton, J. M. (2007). Introduction: Understanding and dealing with organizational survey nonresponse. Organizational Research Methods, 10(2), 195-209. https://doi.org/10.1177/ 1094428106294693

Rupp, D. E., \& Mallory, D. B. (2015). Corporate social responsibility: Psychological, person-centric, and progressing. Annual Review of Organizational Psychology and Organizational Behavior, 2, 211236. https://doi.org/10.1146/annurev-orgpsych-032414-111505

Rupp, D. E., Shao, R., Skarlicki, D. P., Paddock, E. L., Kim, T., \& Nadisic, T. (2018). Corporate social responsibility and employee engagement: The moderating role of CSR-specific relative autonomy and individualism. Journal of Organizational Behavior, 39(5), 559-579. https://doi.org/10.1002/job.2282

Vizcanno, F. V., Martin, S. L., Cardenas, J. J., \& Cardenas, M. (2021). Employees' attitudes toward corporate social responsibility programs: The influence of corporate frugality and polychronic organizational capabilities. Journal of Business Research, 124, 538-546. https://doi.org/10.1016/j.jbusres.2020.11.016

Vlachos, P. A., Panagopoulos, N. G., Bachrach, D. G., \& Morgeson, F. P. (2017). The effects of managerial and employee attributions for corporate social responsibility initiatives. Journal of Organizational Behavior, 38(7), 1111-1129. https://doi.org/10.1002/job.2189

Wicker, A. W. (1969). Attitudes versus actions: The relationship of verbal and overt behavioral responses to attitude objects. Journal of Social Issues, 25, 41-78. https://doi.org/10.1111/j.1540-4560. 1969.tb00619.x

World Business Council for Sustainable Development. (2001). The business case for sustainable development. World Business Council for Sustainable Development. https://www.sustainability-repor ts.com/titel-153/

Xiao, M., Cooke, F. L., Xu, J., \& Bian, H. (2020). To what extent is corporate social responsibility part of human resource management in the Chinese context? A review of literature and future research directions. Human Resource Management Review, 30(4). https://doi.org/10.1016/j.hrmr.2019.100726

Zhao, X., Wu, C., Chen, C. C., \& Zhou, Z. (2020). The influence of corporate social responsibility on incumbent employees: A Metaanalytic investigation of the mediating and moderating mechanisms. Journal of Management, $X X(\mathrm{X}), 1-33$. https://doi.org/10. $1177 / 0149206320946108$

Publisher's Note Springer Nature remains neutral with regard to jurisdictional claims in published maps and institutional affiliations. 